\title{
Sequential Evaluation of Pancreato-Biliary Findings in a Case with IgG4-Associated Cholangiopathy and Autoimmune Pancreatitis during Corticosteroid Treatment
}

\author{
İsmail Şerifoğlu ${ }^{1}$, İbrahim İlker Öz ${ }^{1}$, Yücel Üstündağ ${ }^{2}$, Sevil Uygun İlikhan³ ${ }^{3}$ Özlem Tokgöz \\ ${ }^{1}$ Department of Radiology, Bülent Ecevit University School of Medicine, Zonguldak, Turkey \\ ${ }^{2}$ Department of Gastroenterology, Bülent Ecevit University School of Medicine, Zonguldak, Turkey \\ ${ }^{3}$ Department of Internal Medicine, Bülent Ecevit University School of Medicine, Zonguldak, Turkey \\ ${ }^{4}$ Department of Radiology, Antalya Training and Research Hospital, Antalya, Turkey
}

Background: Immunoglobulin G4 (IgG4)-associated diseases mostly involve the pancreatico-biliary tree and pancreatic parenchyma. This disease complex is characterized by marked response to corticosteroid therapy and response to steroids is incorporated in the diagnostic algorithm of $\mathrm{IgG} 4$ associated diseases. However, there is much unknown about the sequences and duration of healing during the corticosteroid therapy in the literature.

Case Report: In this case report, we report a young male patient with IgG4 associated extrahepatic biliary stricture and autoimmune pancreatitis successfully treated with corticosteroids. Recovery in the laboratory and radiologi- cal findings seemed to correlate well with the decrease in serum IgG4 levels in this patient. We also discussed sequences and the duration of healing in the pancreaticobiliary tree and pancreatic parenchymal manifestations in this case report.

Conclusion: There is a gap in our knowledge about the evaluation of response criteria after steroid trial with regard to the duration and sequences of healing in the pancreaticobiliary involvement in diagnosing IgG4-related biliary and pancreatic diseases.

Keywords: Autoimmune pancreatitis, corticosteroid, IgG4 associated diseases, IgG4, imaging
Corticosteroids are the mainstay of treatment for IgG4-associated diseases including autoimmune pancreatitis, autoimmune cholangiopathy (Sclerosing cholangitis/cholecystitis), salivary/lacrimal gland involvement (Sclerosing sialadenitis/ dacryoadenitis) and retroperitoneal fibrosis (1-4). In clinical practice, these patients mostly present with pancreaticobiliary system involvement such as signs and symptoms of autoimmune pancreatitis, extrahepatic and or intrahepatic biliary strictures (4). Response to steroids is included in the diagnostic algorithm of IgG4-associated diseases. According to the International Consensus Diagnostic Criteria (ICDC) for autoimmune pancreatitis (AIP), rapid (less than 2 weeks) radiologically demonstrable resolution or marked improvement in pancreatic/extrapancreatic manifestations with corticosteroid treatment is accepted as one of the main diagnostic criteria (5).
As far as we know, there are scarce data about which of these organ involvements heals first with corticosteroid treatment and at what point this happens. Herein, we report a patient with IgG4-associated cholangiopathy and autoimmune pancreatitis successfully treated with corticosteroids. We also discuss the sequences and timing of morphologic recovery in the pancreaticobiliary tree and pancreatic organ manifestations in this patient.

\section{CASE PRESENTATION}

A 43 year-old man was admitted to our Gastroenterology Department with jaundice and epigastric dull pain. There was no fever or pruritis. His previous medical history revealed that 

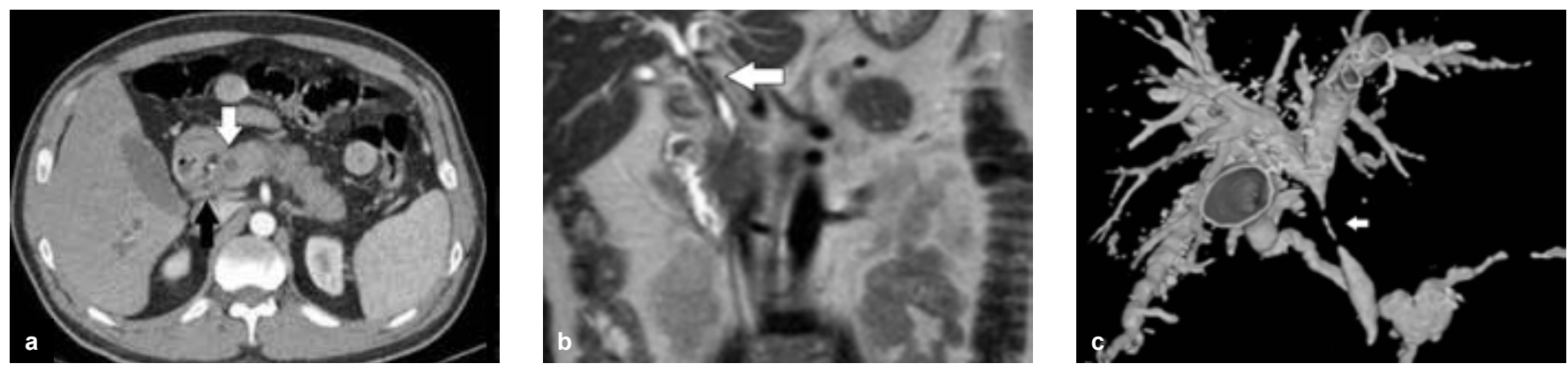

FIG. 1 a-c. Upper Abdominal CT shows diffuse enlargement of pancreas, increased wall thickness in the CBD (black arrow) and cystic dilatation of the pancreatic duct (white arrow) (a), coronal T2-weighted MR image shows increased wall thickness in the CBD (white arrow) (b), 3D reformatted MRCP image shows segmental stricture in the CBD, cystic dilatation of the pancreatic channel with mildly irregular outline is also seen (c).

he has maturity onset diabetes mellitus, ischemic heart disease; his medical treatment consisted of acetyl salicylic acid tablet $100 \mathrm{mg} /$ day (Coraspin, Bayer; Leverkusen, Germany), clopidogrel $75 \mathrm{mg} /$ day (Plavix, Sanofi Winthrop Industrie; Paris, France), metformin 1 gr/day (Glucophage, Merck; West Drayton, UK), bisoprolol 5 mg/day (Concor, Daiichi Sankyo; Tokyo, Japan), ramipril $5 \mathrm{mg}$ /day (Delix, Sanofi-Aventis; Frankfurt, Germany), and insulin glargine twice a day (Lantus, Sanofi-Aventis; Frankfurt, Germany). There was no past history of inflammatory bowel disease. His physical examination was normal, other than mild right upper quadrant pain with palpation. Laboratory tests showed increased serum levels of liver/biliary and pancreatic enzymes, namely alanine aminotransferase $166 \mathrm{IU} / \mathrm{mL}$, aspartate aminotransferase $75 \mathrm{IU} / \mathrm{mL}$, alkaline phosphatase $258 \mathrm{IU} / \mathrm{mL}$, gamma glutamyl transferase $380 \mathrm{IU} / \mathrm{mL}$, total bilirubin $2 \mathrm{mg} / \mathrm{dL}$, direct bilirubin $1.5 \mathrm{mg} /$ $\mathrm{dL}$, amylase $489 \mathrm{U} / \mathrm{L}$, and lipase $384 \mathrm{U} / \mathrm{L}$. Serology for serum autoantibodies including anti-nuclear antibody, anti-smooth muscle antibody, anti-liver kidney microsomal antibody, and anti-mitochondrial antibody were all negative. Serum IgA and IgM levels were all within normal limits. Serum IgG4 level was measured as $1400 \mathrm{mg} / \mathrm{dL}$ (normal range $0-125 \mathrm{mg} / \mathrm{dL}$ ). Computed tomography (CT) (Activision 16-row CT scanner, Toshiba Medical Systems; Otawara, Japan) showed stenosis and asymmetrically increased wall thickness in the distal common bile duct (CBD) (Figure 1a). Pancreas parenchyma was completely edematous and diffusely enlarged. Main pancreatic duct (MPD) was shown to have cystic dilatation at the head region for a few millimeters while the rest of MPD was mildly irregular and dilated. Magnetic resonance imaging (MRI) (1.5 T Gyroscan Intera Master, Philips Medical Systems; DA Best, Netherlands) delineated similar findings to CT (Figure 1b); in addition, a clearly strictured distal CBD was documented on MR cholangio-pancreatography (MRCP) (Figure 1c). IgG4associated cholangiopathy and autoimmune pancreatitis was diagnosed in our case and he was given orally administered $30 \mathrm{mg} /$ day methylprednisolone (Prednol, Mustafa Nevzat İlaç

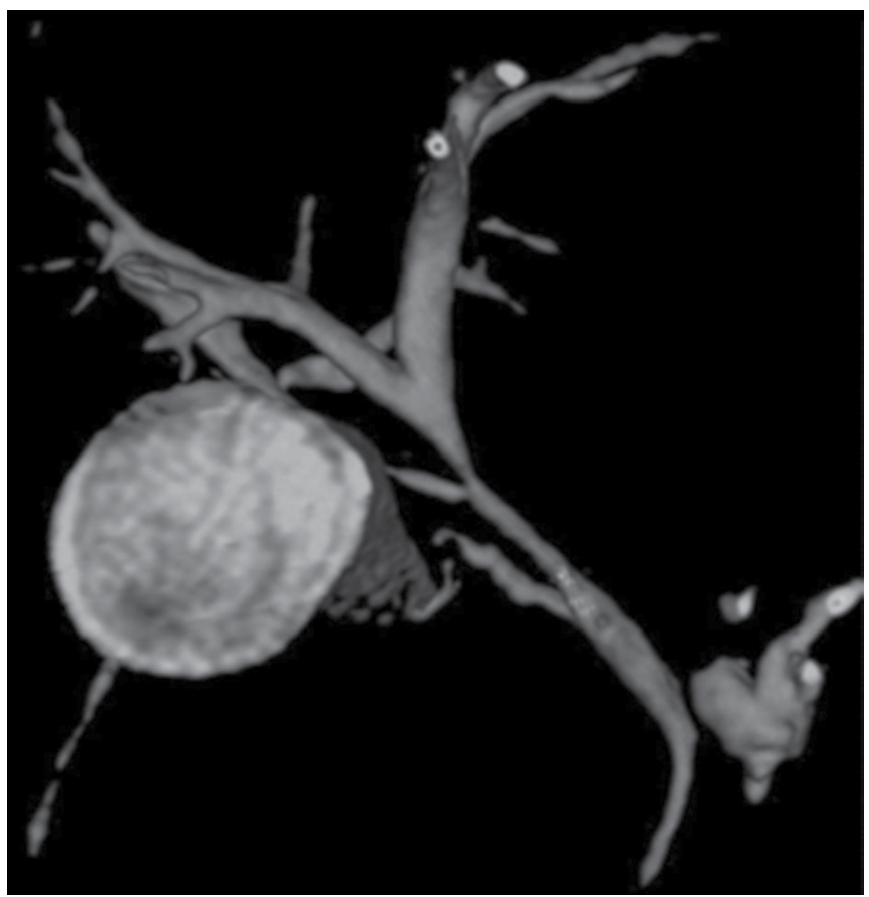

FIG. 2. 3D re-formatted MRCP image after 10 days of treatment shows marked recovery of stenosis in the CBD. Cystic dilatation of the proximal major pancreatic duct persisted.

Sanayii A.Ş.; İstanbul, Turkey). His illness dramatically responded to this treatment and complaints such as epigastric pain and jaundice resolved within 5 and 7 days, respectively. On the $10^{\text {th }}$ day, we repeated the MRCP examination which clearly showed complete clearance of distal CBD stricture and the associated proximal CBD dilatation. However, pancreatic parenchymal and ductal findings persisted on MRCP (Figure 2). Serum IgG4 level at that time was found to be 600 $\mathrm{mg} / \mathrm{dL}$. At the $2^{\text {nd }}$ month of corticosteroid treatment, serum IgG4 level decreased to $200 \mathrm{mg} / \mathrm{dL}$ and on MRCP imaging there was minimal regression in the irregularity of MPC and pancreatic enlargement. Finally, at the end of 3 months of treatment, serum IgG4 level returned to normal $(114 \mathrm{mg} / \mathrm{dL})$ 

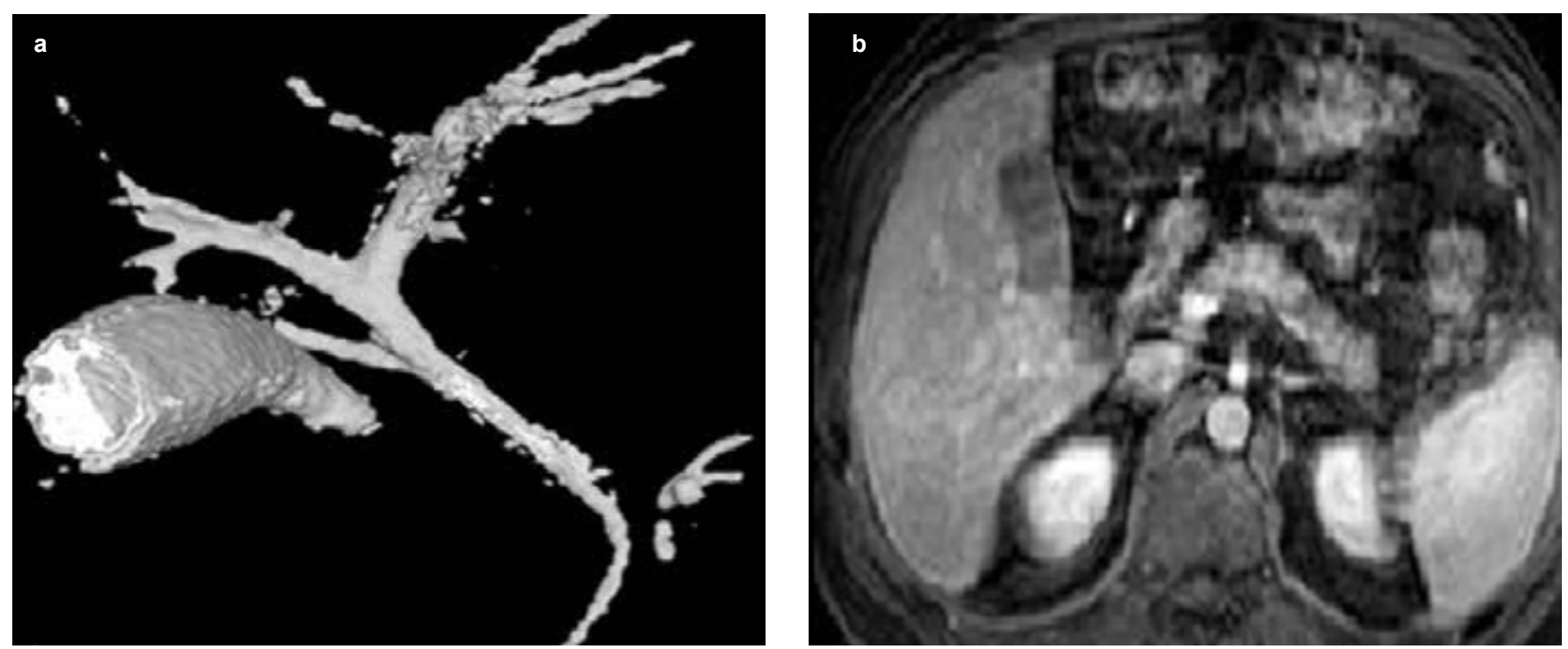

FIG. 3 a-b. 3D MRCP image shows complete resolution of the cystic dilatation in the main pancreatic channel with normally appearing CBD (a), in the contrast-enhanced MR image taken 3 months after corticosteroid treatment, enlargement of the pancreas parenchyma completely disappeared (b).

and pancreaticobiliary tree and pancreatic parenchyma were completely normal on MRCP imaging. Interestingly, small segmentary dilatation at the proximal MPD disappeared completely (Figure $3 \mathrm{a}, \mathrm{b}$ ). Informed consent form was obtained from the patient.

\section{DISCUSSION}

IgG4-associated cholangiopathy most commonly presents with signs and symptoms of acute pancreatitis and cholestasis, as in the present case (4). Although we cannot completely exclude the presence of peripheral IgG4 cholangiopathy, radiological findings were consistent with sclerosing cholangitis, predominantly affecting distal CBD in our case. Most patients with IgG4-associated cholangiopathy have autoimmune pancreatitis, which was also diagnosed in our case on the basis of typical radiological findings such as diffuse enlargement of the pancreas and irregular narrowing of the MPD $(6,7)$. Although it was noticeably an atypical finding, mildly dilated proximal MPD in this case was thought to be related to IgG4-associated disease, since it disappeared completely under corticosteroid treatment. High serum levels of IgG4, the presence of type 1 autoimmune pancreatitis (diffuse type) and corticosteroid responsiveness easily helped us to exclude other possible diseases, such as primary sclerosing cholangitis, cholangiocarcinoma, follicular cholangitis, as the differential diagnosis of this case $(6,7)$. Moreover, histological detection of $\mathrm{IgG} 4^{+}$plasma cells is usually unnecessary for cases like ours, showing typical radiological findings and high serum levels of IgG4. It was reported that high serum IgG4 level (median $663 \mathrm{mg} / \mathrm{dL}$ ) is specific for IgG4-related disease (4).

The recovery of radiological findings within 2 weeks of corticosteroid treatment was accepted as one of the diagnostic criteria for IgG4-related autoimmune pancreatitis by the International Association of Pancreatology (5). Japanese consensus guidelines for the management of autoimmune pancreatitis also emphasize healing of radiologic findings in AIP in 1-2 weeks after the start of steroid therapy (8). This recovery was reported to correlate well with a decrease in the serum levels of IgG4 $(6,7)$. However, in these consensus reports, it is not clear how much resolution in these findings is enough to accept as diagnostic criteria. Moreover, there are very scarce data regarding the sequences of radiologic recovery, and whether or not radiological and clinical sequences correlated with the decrease in serum IgG4 levels are unknown as well. One of the reports indicated that the normalization of pancreatic enlargement in 34 patients with IgG4-associated sclerosing cholangitis occurred after one month of steroid treatment (9). In this study, the authors did not mention the timing of recovery of biliary findings in their cases. Another report indicated that only 2 weeks of steroid treatment induced an improvement in the pancreatic morphology in a case with mass-forming IgG4-related autoimmune pancreatitis (10). In our case, after 10 days of corticosteroid treatment, CBD involvement was the first to disappear completely on MRI and it took a couple of months to see a similar complete response in the pancreatic parenchyma and MPD. Laboratory improvement was also complete after 2 weeks of methylprednisolone treatment. Another interesting finding in our case was that pancreatic findings disappeared completely only after serum 
IgG4 levels decreased to near normal values. Naturally, it is difficult to explain the sequence of healing which occurred first in the biliary tree and then the pancreatic parenchyma and MPD. However, it can be predicted that the number of $\operatorname{IgG4}^{+}$ plasma cells in the pancreas is much higher than the number of IgG4 $4^{+}$plasma cells infiltrating the wall of CBD. Thus, it is reasonable to see resolution first in the biliary tree rather than in the pancreas itself in the setting of IgG4-associated cholangiopathy and autoimmune pancreatitis.

We believe that there is a gap in our knowledge about criteria for the evaluation of response after a steroid trial with regard to the timing and sequence of healing in the pancreaticobiliary involvement in the diagnostic process of IgG4related biliary and pancreatic diseases. There is an absolute need for more data on this subject which can be provided by case series and original investigations. This information is really important because we can use these data to design appropriate follow-up of these patients. Furthermore, these data can be useful for timely differentiation of pancreatic malignancy from an auto-inflammatory pancreatic process.

\section{Ethics Committee Approval: N/A.}

Informed Consent: Written informed consent was obtained from patient who participated in this study.

Peer-review: Externally peer-reviewed.

Author contributions: Concept - İ.Ş., İ.İ.Ö., Y.Ü.; Design - İ.Ş., Y.Ü.; Supervision - Y.Ü.; Resource - Y.Ü., S.U.İ.; Materials Y.Ü., S.U.İ.; Data Collection and/or Processing - İ.Ş., İ.İ.Ö., S.U.İ.; Analysis and/or Interpretation - Y.Ü., Ö.T.; Literature Search - İ.Ş., İ.İ.Ö., Ö.T.; Writing - İ.Ş., Y.Ü.; Critical Reviews - Y.Ü., Ö.T.

Conflict of Interest: No conflict of interest was declared by the authors.

Financial Disclosure: The authors declared that this study has received no financial support.

\section{REFERENCES}

1. Kitagawa S, Zen Y, Harada K, Sasaki M, Sato Y, Minato H , et al. Abundant IgG4-positive plasma cell infiltration characterizes chronic sclerosing sialadenitis (Küttner's tumor). Am J Surg Pathol 2005;29:783-91. [Crossref]

2. Yamamoto M, Harada S, Ohara M, Suzuki C, Naishiro Y, Yamamoto $\mathrm{H}$, et al. Clinical and pathological differences between Mikulicz's disease and Sjögren's syndrome. Rheumatology 2005;44227-34. [Crossref]

3. Zen Y, Onodera M, Inoue D, Kitao A, Matsui O, Nohara T, et al. Retroperitoneal fibrosis: a clinicopathologic study with respect to immunoglobulin G4. Am J Surg Pathol 2009; 33:18339. [Crossref]

4. Hamano H, Kawa S, Horiuchi A, Unno H, Furuya N, Akamatsu $\mathrm{T}$, et al. High serum IgG4 concentrations in patients with sclerosing pancreatitis. $N$ Engl J Med 2001;344:732-8. [Crossref]

5. Shimosegawa T, Chari ST, Frulloni L, Kamisawa T, Kawa S, Mino-Kenudson $\mathrm{M}$, et al. International consensus diagnostic criteria for autoimmune pancreatitis: guidelines of the International Association of Pancreatology. Pancreas 2011;40:352-8. [Crossref]

6. Takuma K, Kamisawa T, Igarashi Y. Autoimmune pancreatitis and IgG4-releated sclerosing cholangitis. Curr Opin Rheumatol 2011;23:80- 7. [Crossref]

7. Okazaki K, Tomiyama T, Mitsuyama T, Sumimoto K,Uchida K. Diagnosis and classification of autoimmune pancreatitis. Autoimmun Rev 2014;13:451-8. [Crossref]

8. Kamisawa T, Okazaki K, Kawa S, Shimosegawa T, Tanaka M. Japanese consensus guidelines for management of autoimmune pancreatitis: III. Treatment and prognosis of AIP. J Gastroenetrol 2010;45:471-7. [Crossref]

9. Kamisawa T, Okamoto A, Wakabayashi T, Watanabe T, Sawabu N. Appropriate steroid therapy for autoimmune pancreatitis based on long-term outcome. Scand $J$ Gastroenterol 2008;43:609-13. [Crossref]

10. Shimizu S, Naitoh I, Nakazawa T, Hayashi K, Okumura F, Miyabe $\mathrm{K}$, et al. A case of autoimmune pancreatitis showing narrowing of the main pancreatic duct after cessation of steroid therapy in the clinical course. Intern Med 2012;51:2135-40. [Crossref] 